\title{
MAGNETIC RESONANCE IMAGING IN THYROID EYE DISEASE
}

\author{
C. C. BAILEY ${ }^{1}$, J. KABALA ${ }^{2}$, R. LAITT ${ }^{2}$, P. GODDARD ${ }^{2}$, H. B. HOH ${ }^{1}$, M. J. POTTS ${ }^{1}$ and \\ R. A. HARRAD ${ }^{1}$ \\ Bristol
}

\begin{abstract}
SUMMARY
We examined 25 patients with thyroid eye disease, using both the STIR (Short Tau Inversion Recovery) sequence and cine MRI techniques. A number of characteristic features can be seen on the cine MRI. There is muscle enlargement with restriction of movement and, in the burnt-out phase of the disease, reduced elasticity of the muscles is manifest as their failure to stretch on eye movement. This is in contrast to the active phase of the disease, where although the muscles are enlarged, muscle stretching is clearly visible. The STIR sequence gives an assessment of muscle water content, and hence a high signal is seen in active disease. Combining these techniques is useful in assessing the level of disease activity in thyroid eye disease, and helps in planning further management.
\end{abstract}

Significant thyroid eye disease occurs in $2-7 \%$ of cases of Graves' disease. ${ }^{1}$ There appear to be two stages in the disease process: an active phase characterised by inflammation with a lymphocytic infiltrate of the retro-orbital tissues, and a quiescent (burnt-out) phase..$^{2-5}$ In the latter, fibrosis has occurred, but there is no active inflammation.

Magnetic resonance imaging (MRI) has several advantages over computed tomography (CT) in the assessment of thyroid eye disease There is excellent soft tissue differentiation; no ionising radiation is used, so multiple sequences are possible and no patient repositioning is required to take scans in different planes. ${ }^{6,7}$ Those features characteristic of thyroid eye disease seen on CT such as muscle enlargement with relative sparing of the tendon, bowing of the medial wall of the orbit, and muscular compression at the orbital apex in cases of optic neuropathy, ${ }^{4}$ will also be seen on MRI, but with better tissue contrast. By using different scanning techniques, further information on disease activity can be obtained.

From: ${ }^{1}$ Bristol Eye Hospital, Bristol; ${ }^{2}$ Department of Radiology, Bristol Royal Infirmary, Bristol, UK.

Correspondence to: C. C. Bailey, Bristol Eye Hospital, Lower Maudlin Street, Bristol BS1 2LX, UK.
We have used both the STIR (Short Tau Inversion Recovery) sequence and cine MRI techniques, ${ }^{8,9}$ to examine 25 patients with thyroid eye disease. The STIR sequence ${ }^{10}$ suppresses the signal from orbital fat, and enhances the signal from water-containing tissues. Active inflammation is associated with oedema within the tissue, and will give a bright signal on the STIR sequence, which can be used to assess the degree of inflammation within the extraocular muscles. The cine MRI technique takes multiple static orbital images while the patient fixates a series of targets across the visual field, and allows an assessment of muscle contractility.

\section{METHOD}

A Siemens Magnetom Impact MRI scanner was used. In the STIR sequence (TR/TE/TI 3000/20/150), images were taken in the coronal plane. For the cine MRI technique, a FISP two-dimensional sequence was used. A strip of card with six separate lines set $30^{\circ}$ apart was suspended in front of the patient and aligned either transversely or sagittally depending on the eye movement of interest. The patient was asked to look sequentially at each mark on the paper, and a scan was performed in each position. Each image took 15 seconds to acquire. After the patient had fixated all six points, the procedure was repeated in reverse order. The images generated were then displayed as a continuous video loop. ${ }^{9}$

Each patient was examined clinically, and their disease graded in accordance with the Werner and Mourits classifications. ${ }^{11,12}$

The static scans were studied and the length of the medial recti in abduction and adduction were measured. In addition the maximum width of the rectus muscles was measured from the coronal scans. The Signal Intensity Ratio (SIR) of each muscle was measured, by comparing the STIR signal from the extraocular muscle (pixel analysis of its brightness) with that from the temporalis muscle on that side, and expressing the results as a ratio. These findings 


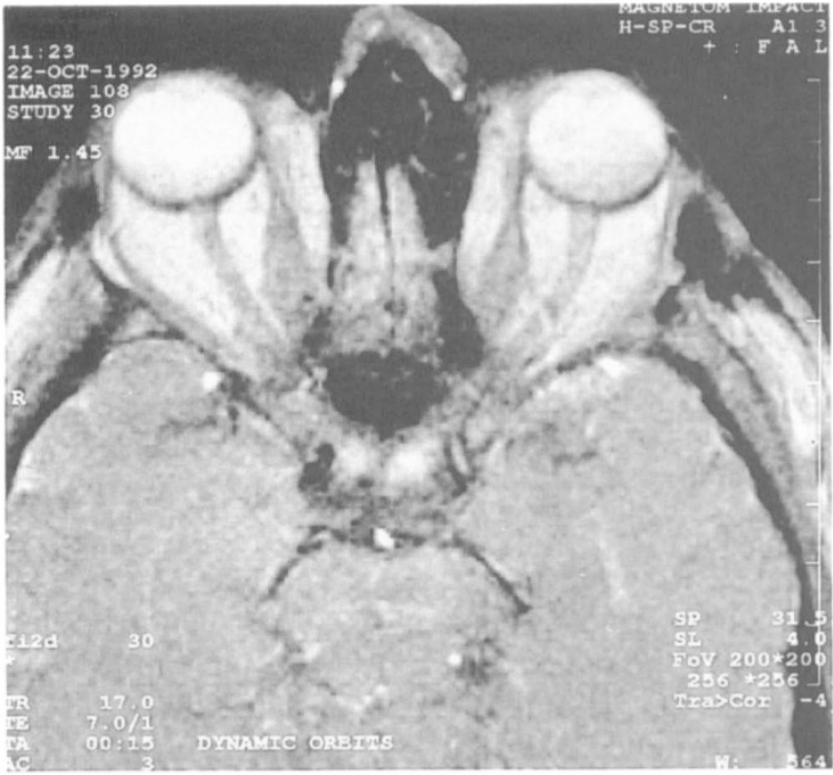

(a)

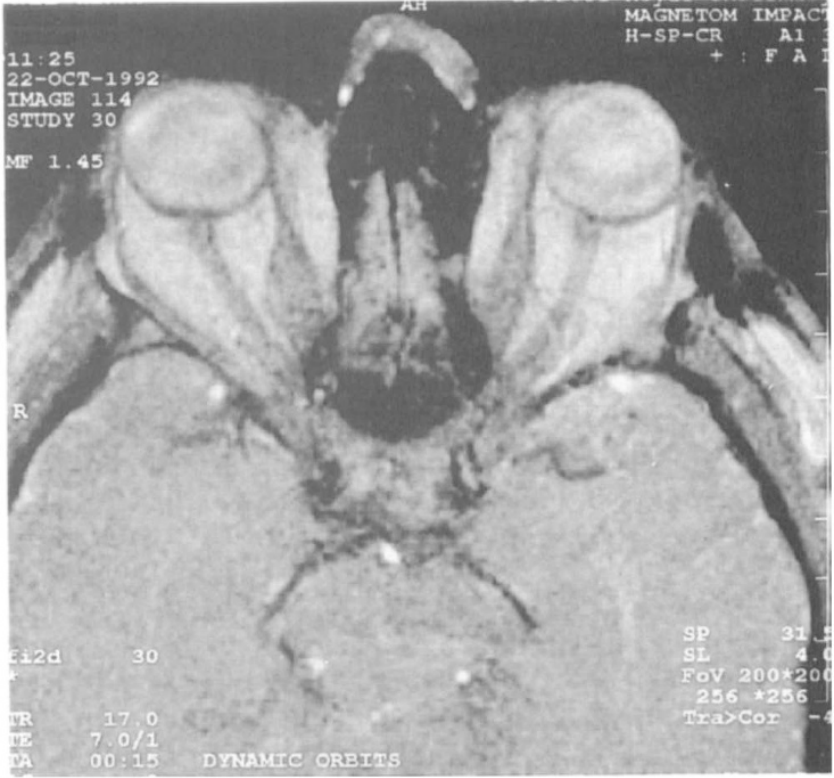

(b)

Fig. 1. Quiescent (burnt-out) thyroid eye disease. Scans in abduction (a) and adduction (b) of the right eye. The right eye is seen on the left-hand side of the picture in keeping with scanning convention. Note how little the horizontal recti change shape or stretch between abduction and adduction, indicative of reduced muscle elasticity and fibrosis.

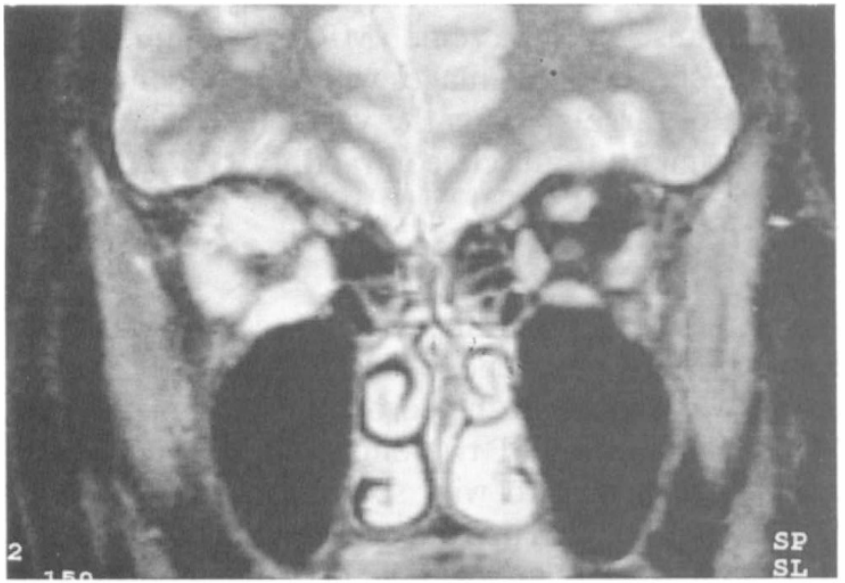

Fig. 2. STIR sequence MRI in active thyroid eye disease. There is enlargement of the extraocular muscles, particularly in the right eye, which give a bright signal on the STIR sequence, indicating a high water content and active inflammation.

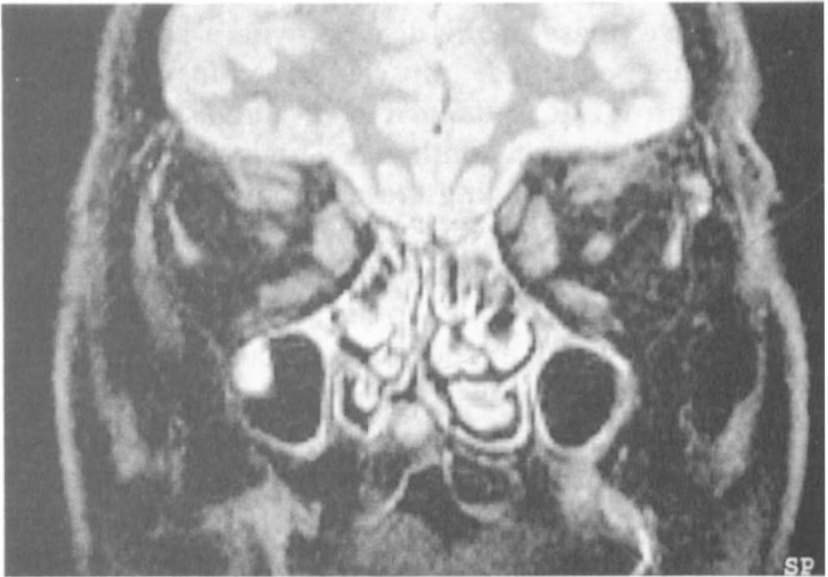

Fig. 3. STIR sequence MRI in burnt-out thyroid eye disease. Although the extraocular muscles remain enlarged, they give a low signal on the STIR sequence, suggesting that they are not actively inflamed. There is an inflammatory polyp in the right maxillary antrum.

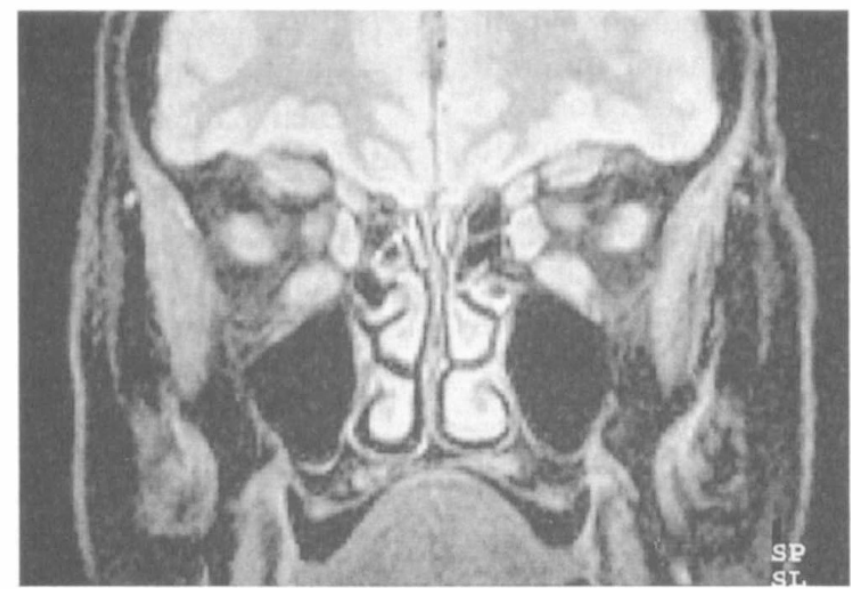

Fig. 4. STIR sequence MRI in early active thyroid eye disease showing a halo of increased signal intensity around the muscles, particularly around the medial recti. This probably represents increased inflammation in the muscle sheath. 
were compared with the level of disease activity graded clinically. ${ }^{10}$

\section{RESULTS}

Using the cine MRI technique a number of characteristic features could be seen. Enlargement of the extraocular muscles with relative sparing of the tendons was observed. There was impaired ocular motility in both the active and burnt-out phases of the disease, but in the burnt-out phase muscle elasticity was reduced, manifesting as failure of the muscles to stretch on eye movement (Fig. 1). In contrast, muscle stretching was clearly visible in the active phase of the disease. The normal wide range of movement of the optic nerve within the orbital fat ${ }^{13}$ was impaired, due to either stretching or splinting of the nerve by the enlarged muscles. In cases with optic neuropathy, crowding at the orbital apex by the enlarged extraocular muscles could be seen, with an enlarged CSF cuff around the optic nerve more anteriorly.

Using the STIR sequence we found early active disease to be associated with a high SIR and relatively little muscle enlargement, whereas in advanced active disease considerable muscle enlargement was associated with a high SIR (Fig. 2). ${ }^{10}$ In burnt-out disease, although muscle enlargement persisted there was a lower SIR (Fig. 3). Several cases showed increased signal intensity within the orbital fat as well as the extraocular muscles. Another feature seen in many cases of early disease was a halo of increased signal intensity around the muscle (Fig. 4). This radiological feature has not been described previously, and may represent increased oedema and inflammation in the sheath of the muscle. Pathological studies have indicated that the fibroblasts may be the initial antigenic target in thyroid-related ophthalmopathy. These are more abundant in the connective tissue around the muscle, and they produce glycosaminoglycans in response to the immune attack. These glycosaminoglycans osmotically attract water resulting in interstitial oedema. At this early stage the muscle fibres themselves are thought not to be affected. ${ }^{4,5,14,15}$

\section{DISCUSSION}

MRI has many advantages over CT in the assessment of thyroid eye disease. There is excellent soft tissue differentiation, no ionising radiation is used, and the STIR sequence can give an assessment of the level of active inflammation in the muscles. ${ }^{6,10}$ The cine MRI technique gives an assessment of muscle contractility. ${ }^{8,}$ Reduced muscle elasticity is seen in the quiescent phase, whereas muscle stretching is clearly visible in the active phase of the disease. These techniques can lead to the detection of subclinical disease, and can demonstrate the natural history of the disease process by revealing the sequence of involvement of the extraocular muscles. Squint surgery should be delayed if unexpected activity of disease is noted in one or more muscles, and the use of botulinum toxin should be avoided if the muscles are clearly fibrotic and rigid. ${ }^{16}$ Moreover, immunosuppressive therapy should only be considered in cases with active disease, and the degree of inflammation of the muscles on the STIR sequence, in conjunction with the clinical grading of disease activity, can give an indication of the likelihood of benefit from immunosuppressive therapy.

We feel that these techniques are an invaluable adjunct to clinical examination in thyroid eye disease. They help to assess the level of disease activity, aid in the planning of appropriate treatment, and provide information about the natural history of the disease.

Key words: Magnetic resonance imaging, Thyroid eye disease.

\section{REFERENCES}

1. Gorman CA. Ophthalmology in Graves' disease. [editorial]. N Engl J Med 1983;308:453-5.

2. Weetman AP, McGregor AM, Hall S. Ocular manifestations of Graves' disease: a review. J R Soc Med 1984;77:936-42.

3. Sergott RC, Feldberg NT, Savino PJ, et al. E-rosette formation in Graves' ophthalmopathy. Invest Ophthalmol Vis Sci 1979;18:1245-51.

4. Trokel SL, Jakobiec FA. Correlation of CT scanning and pathologic features of ophthalmic Graves' disease. Ophthalmology 1981;88:553-64.

5. Fells P, Kousoulides L, Pappa A, Munro P, Lawson J. Extraocular muscle problems in thyroid eye disease. Eye 1994;8:497-505.

6. Han JS, Benson JE, Bonstelle CT, Alfidi RJ, Kaufman $\mathrm{B}$, Levine M. Magnetic resonance imaging of the orbit. Radiology 1984;150:755-9.

7. Jay WM. Advances in magnetic resonance imaging. Am J Ophthalmol 1989;108:592-6.

8. Cadera W, Viirre E, Karlik S. Cine magnetic resonance imaging of ocular motility. J Pediatr Ophthalmol Strabismus 1991;29:120-2.

9. Bailey CC, Kabala J, Laitt R, Hoh HB, Goddard P, Potts MJ, Harrad RA. Cine magnetic resonance imaging of eye movements. Eye 1993;7:691-3.

10. Hoh HB, Laitt RD, Wakeley C, Kabala J, Goddard P, Potts MJ, Harrad RA. The STIR sequence MRI in the assessment of extraocular muscles in thyroid eye disease. Eye 1994;8:506-10.

11. Werner SC. Modification of the classification of eye changes of Graves' disease. Am J Ophthalmol 1977;83: 725-7.

12. Mourits MP, Koornneef LK, Wiersinga WM, et al. Clinical criteria for the assessment of disease activity in Graves' ophthalmology: a novel approach. $\mathrm{Br}$ J Ophthalmol 1989;73:639-44.

13. Smiddy WE, Michels RG, Kumar AJ. Magnetic resonance imaging of retrobulbar changes in optic nerve position with eye movement. Am J Ophthalmol 1989;107:82-3.

14. Kroll A, Kuwabara T. Dysthyroid ocular myopathy: anatomy, histology and electron microscopy. Arch Ophthalmol 1966;76:244-57.

15. Bahn RS, Heufelder AE. Pathogenesis of Graves' ophthalmopathy. N Engl J Med 1993;329:1468-75.

16. Lyons CJ, Vickers SF, Lee JP. Botulinum toxin therapy in dysthyroid strabismus. Eye 1990;4:538-40. 